\title{
The Application of Fuzzy Reasoning in Cement Particle Size Soft Sensor
}

\author{
Weilei Luan, Qingjin Meng, Tao Shen* \\ School of Electrical Engineering \\ University of Jinan \\ Jinan, China \\ e-mail: luanweilei@163.com,jdmqj@sohu.com,cse_st@ujn.edu.cn( corresponding author)
}

\begin{abstract}
In the cement production process, cement quality is the most important indicator. One way to measure the quality of the cement is the cement particle size. Only the cement particle size is symmetrical and the specific surface area could reach the national standard that this kind of cement is qualified products. In actual production, the specific surface area is tested in laboratory through experiment. This process takes a long time, and the instantaneous value of specific surface area can not be got. This paper, auxiliary variables coming from the practical production site are used to get an instantaneous value through fuzzy control algorithm and other rules. $\mathrm{VC}++6.0$ is used to program this algorithm. After a long period of actual operation, this method is proved to be effective, the instantaneous specific surface area is got and the precision is acceptable. This could guide the operator to change parameters of the equipment timely. This could be widely used and is easy to spread.
\end{abstract}

Keywords-cement particle size; specific surface area; fuzzy reasoning; soft measurement

\section{INTRODUCTION}

In the cement production process, cement particle size is the most important indicator. It is used to detect the quality of cement. The cement particle size is reflected by specific surface area of cement. The definition of specific surface area is the whole superficial area of the unit quality cement powder. Generally, its' unit is square centimeters per gram $(\mathrm{cm} 2 / \mathrm{g})$. [1]The specific surface area of cement can't be measured at all time in the whole production period. Its' value can only be got through the result tested by the chemical laboratory. Therefore, long delay is unavoidable and operators can not adjust a variety of equipments in time.

At present, in domestic and abroad the means of measurement of cement of particle size are offline and online. Of course, the online method is much more advanced than the offline method, but the equipment of online method is too expensive for many of the cement industries, and the precision is always frustrated. So most industries test the cement particle size in chemical laboratory and the frequency is once an hour. The lab assistant takes only a few grams as the sample, obviously, this little sample couldn't represent the whole cement in the whole period. The long interval is a great disadvantage, the operator can't ensure the quality of the cement quality in this period is all qualified. This leads to a serious shortage of production guidance and then increasing the probability of substandard products. This method can not meet the requirements of modern technology development and production. The soft measurement can solve both of the problems [2], the cement particle size can reflect in the monitor in the center control room in all time.

\section{CEMENT GRINDING PROCEss}

According to a certain proportion, some kinds of raw materials are mixed and went into the $\mathrm{V}$-separator through a lifting motor. The material are affected by the gravity and the speed of the circulating fan, then the big particle going down into the weighing bin. After the grinding of roller press machine, the whole materials are blended together. The small particles driven by the fan enter into the ball mill, after the grinding of the ball mill, the material are lifted into the classifiers. The qualified material are transferred into the cement silo by a lifting machine, at the same time, the unqualified material are put into the ball mill again for regrinding. Obviously, this whole process flow could be separated into two loops. The flow is showed in Figure.1.

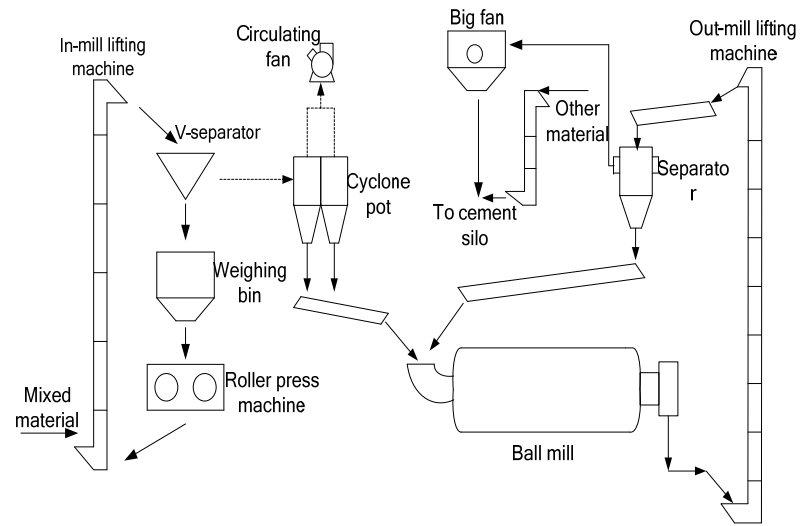

Figure 1. Cement Production Process

In the cement production process cyclone separator machine has the most important effect on the cement particle size. Cyclone separator has a speed control motor and two speed-reduce motors, these motors drive the blades rotating, so the cement is classified. The smaller particles you want to get, the faster speed of the blades is needed, in this way, the contact time for the cement and the blades will be much longer. The unacceptable cement will be divided with the wind flow. The big particles fall into the ball mill because of its own gravity. The acceptable cement goes with the airflow into the big collector. When the wind flow and the cement is divided, the cement is collected into the cement silo. 


\section{DESIGN OF THE SOFT MEASUREMENT SYSTEM}

\section{A. Process of Soft Measurement}

First of all, auxiliary variables should be chosen according to the production process, and then some data preprocessing should be made [3,4]. Based on the processed data, a fuzzy controller is designed to achieve the goal of a soft measuring. Finally, in each control period, parameter correction is done. Flow chart of soft measurement is shown in Figure.2.

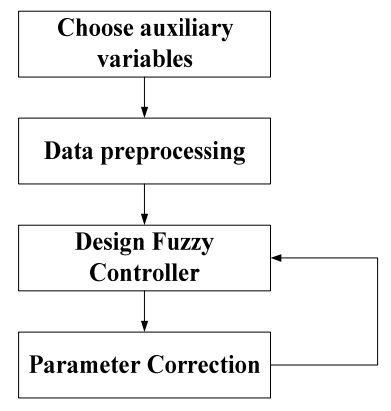

Figure 2. Soft Measurement Flow Chart

\section{B. Factors Affect Cement Particle Size}

- Due to the grinding process, the phenomenon of segregation will occur in the weighing bin. Segregation is due to the uneven particle size of the material in the clinker silo, some material size are massive, while some are in powder form. In the grinding process the secondary separation is occurred, the massive particles comes down last, at this time the roller press runs more efficiently. This is a periodically process, and it has some relationship with the specific surface area.

- The weather condition is another main influence, dry weather differs with the wet weather. When the weather is dry, the material is easy to grind, on the contrary, if the weather is wet, the material is likely to combine together. The specific surface area will be influenced by the weather.

\section{The Selection of Auxiliary Variables}

For the choice of auxiliary variables, first we have to analyze the mechanism of the process of industrial processes, and then we should ensure the correlation variables which have some effects to the results of the soft measurement. Finally, after analyzing the observability and controllability of each variable, we finish the primary election. A good selection of auxiliary variable is essential to form a mature and effective soft measurement control program.

According to the above auxiliary variable selection principle, and combined with practical experience in cement production, we decided to select the out-mill lifting machine current as the most important auxiliary variable. If we don't consider other confounding factors in the cement production, the increase of the separator speed results in the increase of the feedback of the material, the material go back into the ball mill again, the current of the lifting machine rise up.
What's more, the increase or decrease rate of the current also has an effect to the particle size of cement.

\section{Design of Fuzzy Controller}

Based on the auxiliary variables we selected, we decide to use the fuzzy control algorithm to estimate the cement specific surface area. Dual-input single-output structure is selected as the fuzzy controller's structure [5,6]. The current setpoint of the out-mill lifting machine is $d_{r}$, and $d_{1}, d_{2}, \cdots, d_{n}$ are the value of current in a control period. Equation (1) is used to gain an average value of the current.

$$
\bar{d}=\frac{1}{n} \sum_{i=1}^{n} d_{i}
$$

The error of the out-mill lifting machine current and the current setpoint is $e, e c$ is the error rate. $e$ and $e c$ are selected as the input of the fuzzy controller. The change value of the specific surface area $\Delta u(i)$ is the output of the controller. At the time $i$, the error is, $e(i)=d(i)-d_{r}$, error rate is, $e c(i)=e(i)-e(i-1)$, output is, $u_{b}(i)=u(i-1)+\Delta u(i)$.

The variables $e, e c$ and $\Delta u(i)$ are divided into nine fuzzy domains \{negative amass, negative large, negative medium, negative small, zero, positive small, positive medium, positive large, positive amass $\}$, for short $\{\mathrm{NA}, \mathrm{NB}$, NM, NS, ZE, PS PM, PB,PA\}, according to the factual production, $(-30,30)$ is selected as the input and output domain and Gaussian function is used as the membership function, as is shown in Figure.3.

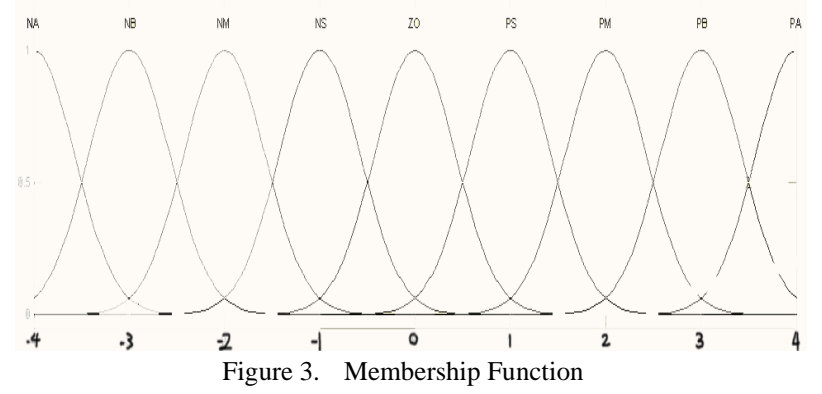

The ascertain of quantization factors. Quantization factors are introduced to realize the obfuscation of data. After repeated testing, the domain of $e$ is $[-4,4]$, the domain of $e c$ is $[-1.2,1.2]$ and $u_{b}$ is $[-20,20]$. So the quantization factors $K_{e}, K_{e c}$ are calculated as (2) and scale factor $K_{u}$ is calculated as (3).

$$
\begin{gathered}
K_{e}=\frac{30}{3.6}=8.33, K_{e c}=\frac{30}{1.2}=25 \\
K_{u}=\frac{1}{20}=0.05
\end{gathered}
$$

The selection of fuzzy control rules are based on expert knowledge and it's a summary of the practical experience of the operator. The rule table is shown in TABLEI. 
TABLE I. TABLE OF FUZZY CONTROL RULES

\begin{tabular}{|c|l|l|l|l|l|l|l|l|l|}
\hline $\begin{array}{c}\text { ec } \\
\text { e }\end{array}$ & NA & NB & $\begin{array}{c}\text { N } \\
\text { M }\end{array}$ & NS & ZO & PS & PM & PB & PA \\
\hline NA & PA & PA & PA & PA & PA & PB & PB & PB & PB \\
\hline NB & PA & PB & PB & PB & PB & PM & PS & ZO & PM \\
\hline NM & PB & PB & PB & PM & PM & PS & ZO & ZO & PS \\
\hline NS & PM & PM & PM & PS & PS & ZO & ZO & NS & ZO \\
\hline ZO & PS & NM & NS & NS & ZE & PS & PM & PB & NS \\
\hline PS & ZO & PS & ZO & ZO & NO & NB & NB & NB & NM \\
\hline PM & NS & ZO & ZO & NO & NM & NB & NB & NB & NB \\
\hline PB & NM & ZO & NS & NM & NB & NB & NB & NB & NA \\
\hline PA & NB & NB & NB & NA & NA & NA & NA & NA & NA \\
\hline
\end{tabular}

The weighted average method is used to make the ambigurious value clear as (4). $\mu_{i}(k)$ is the extent of membership, $u_{i}$ is the domain of $u_{b}$. Finally multiply $U$ by $K_{u}$ we get $u_{b}$ 。

$$
U=\frac{\sum_{i=1}^{n} \mu_{i}(k) u_{j}}{\sum_{j=1}^{n} \mu_{i}(k)}
$$

\section{E. Other Parameters Test Principles}

Due to the material segregation effect, when large materials go into the press roller machine and the current $i_{a}$ is higher, so it's easy to crush. There is much more small powder. This powder flow goes into ball mill and obviously the specific surface area of out-mill cement is much higher. So some minor adjustment $\Delta u a$ should be added to $u_{b}$. After a long time of observation and debugging in cement plant and the rules of $\Delta u a$ are shown in TABLE II.

Similarly, the current of in-silo lifting machine $\Delta i_{b}$ also have an effect on the specific surface area. If $\Delta i_{b}$ rises up, there is more qualified cement goes into the cement silo, and if the current rate is quickly, the specific surface area will decrease. On this condition, $\Delta u b$ is added to $u_{b}$ to compensate for the precision. Based on this principle, the rules are shown in TABLE III.

TABLE II. $\quad \Delta u a$ ADD TO $\boldsymbol{u}_{b}$

\begin{tabular}{|l|l|l|l|l|l|}
\hline NO. & $i_{a}$ & $\Delta u u$ & NO. & $i_{a}$ & $\Delta u u$ \\
\hline 1 & $45<i_{a}<46$ & -5 & 6 & $50<i_{a}<51$ & 1 \\
\hline 2 & $46<i_{a}<47$ & -4 & 7 & $51<i_{a}<52$ & 2 \\
\hline 3 & $47<i_{a}<58$ & -3 & 8 & $52<i_{a}<53$ & 3 \\
\hline 4 & $48<i_{a}<49$ & -2 & 9 & $53<i_{a}<54$ & 4 \\
\hline 5 & $49<i_{a}<50$ & -1 & 10 & $54<i_{a}<55$ & 5 \\
\hline
\end{tabular}

\begin{tabular}{|c|l|l|l|l|l|}
\hline NO. & \multicolumn{1}{|c|}{$i_{b}$} & $\Delta u u$ & NO. & $i_{R}$ & $\Delta u u$ \\
\hline 1 & $46<i_{b}<47$ & 9 & 6 & $51<i_{b}<52$ & -1 \\
\hline 2 & $47<i_{b}<48$ & 7 & 7 & $52<i_{b}<53$ & -3 \\
\hline 3 & $48<i_{b}<49$ & 5 & 8 & $53<i_{b}<54$ & -5 \\
\hline 4 & $49<i_{b}<50$ & 3 & 9 & $54<i_{b}<55$ & -7 \\
\hline 5 & $50<i_{b}<51$ & 1 & 10 & $55<i_{b}<56$ & -9 \\
\hline
\end{tabular}

\section{SYSTEM IMPLEMENTATION AND APPLICATION EFFECT}

\section{A. Software In Use}

Visual $\mathrm{C}++6.0$ software is used as the programming environment and all the algorithm is put into one program. The interface of the software is shown in Figure.4. In this software we could see many variables, such as out-mill lifting machine current, current error rate, the speed of separator, etc. In the production place, there are two kinds of cement, one is PC32.5, and the other is PC42.5. When produce one kind, press the corresponding button, and the specific surface area will be displayed. The software was showed in Fig.5. In this picture the cement type is PC32.5 which is similar to PC42.5.

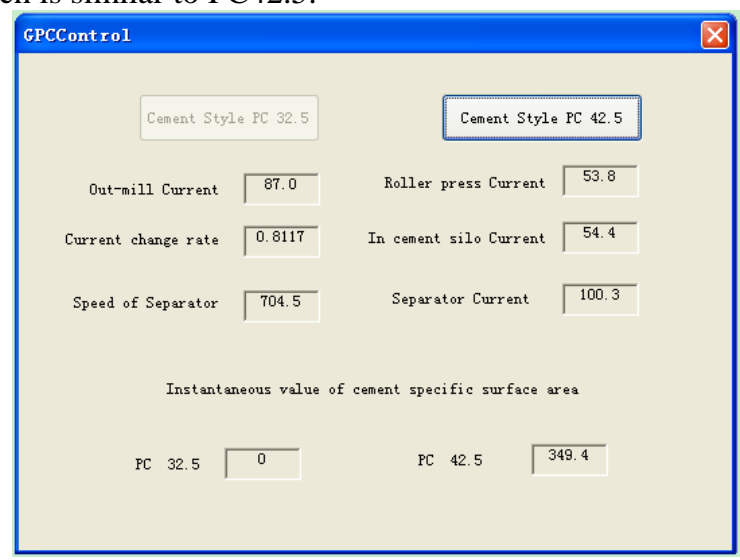

Figure 4. Software In Use

Software is connected to the sever via OPC interface technology. In each sampling period, the software reads the variables from OPC sever and calculate the specific surface area out. The sampling period is 5 minutes. So the operator could know the quality of the cement in time.

\section{B. Software Application Effect Analysis}

After a period of operation in a cement plant, the comparative analysis of specific surface of cement obtained by the software with the laboratory data, the error is less than $5 \%$. The contrast is shown in Figure.5, the full black 
line represents the laboratory data and the dotted line represents the soft measurement data. Obviously, the precision is relatively high, and the calculated value could be a reference value. This could guide the operator change the parameters of equipments, in this way, the quality of cement is increased. What's more, the quality is more stable than before.

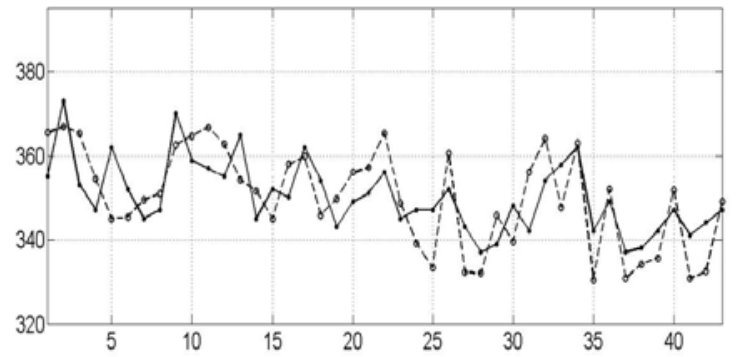

Figure 5. Contrast Between Laboratory Data and Soft Measurement

\section{SUMMARY}

The use of soft measurement to calculate the surface area of cement has important meaning. On the one hand, it could increase the quality of the cement; on the other hand, this is much cheaper than other means. The operator could know the instantaneous quality of the cement, and make some timely changes to the equipments. As a result, the quality is stable, and the stable state is good to all the production process. This software brings cogent benefits to a cement plant in Shandong province.

\section{ACKNOWLEDGMENT}

This work was supported by the Natural Science Foundation of Shandong Province, China (Grant No.ZR2010FM038), and was supported by another Natural Science Foundation of Shandong Province, China (Grant No.ZR2010FM020).

\section{REFERENCES}

[1] Dale P.Bentz, Chiara F.Ferraris, Michael A. Galler, Influence of particle size distributions on yield stress and viscosity of cementflyash pastes, Cement and Concrete Research,vol. 42, pp. 404409,2011.

[2] Marcos J. Arauzo-Bravoa, Jose M.Cano-Izquierdo, Eduardo GomezSanchez, Automatization of a penicillin production process with soft sensors and an adaptive controller based on neuro fuzzy systems, Control Engineering Practice, vol. 12, pp. 1073-1090,2004.

[3] Xiaojia Li, Zhugang Yuan. Soft Sensor of the Material Flow from the New Suspension Preheater Kiln. 2011 3rd International Conference o n Computer and Network Technology (ICCNT 2011), vol.152, pp.557-561,2011.

[4] Yuan Zhugang, Liu Hui. Soft Sensor for Apparent Degree of Calcination in NSP Cement Production Line. In:The 2nd International Conference on Computer and Automation Engineering (ICCAE 2010), vol.1,pp.473-478, 2010.

[5] Jose M.Andujar, Antonio J.Barragan, A methodology to design stable nonlinear fuzzy control systems, Fuzzy Sets and Systems, vol.154,pp. 157-181,2005.

[6] YU Nkhizhnyakov,AA Yuzhakov, Automation of Self-Contained Multiunit Electric Power Stations Based on Fuzzy Control Using Neuron Technologies, Russian Electrical Engineering,vol.82,pp.612$617,2011$. 